\title{
Systematic genome editing of the genes on zebrafish Chromosome 1 by CRISPR / Cas9
}

\author{
Yonghua Sun, ${ }^{1}$ Bo Zhang, ${ }^{2}$ Lingfei Luo, ${ }^{3}$ De-Li Shi, ${ }^{4}$ Han Wang, ${ }^{5}$ Zongbin Cui, ${ }^{1}$ \\ Honghui Huang, ${ }^{3}$ Ying Cao, ${ }^{6}$ Xiaodong Shu, ${ }^{7}$ Wenqing Zhang, ${ }^{8}$ Jianfeng Zhou, ${ }^{9}$ \\ Yun Li, ${ }^{9}$ Jiulin Du, ${ }^{10}$ Qingshun Zhao, ${ }^{11}$ Jun Chen, ${ }^{12}$ Hanbing Zhong, ${ }^{13}$ Tao P. Zhong, ${ }^{14}$ \\ Li Li, ${ }^{3}$ Jing-Wei Xiong, ${ }^{15}$ Jinrong Peng, ${ }^{12}$ Wuhan Xiao, ${ }^{1}$ Jian Zhang, ${ }^{16}$ jihua Yao, ${ }^{17}$ \\ Zhan Yin, ${ }^{1}$ Xianming Mo, ${ }^{18}$ Gang Peng, ${ }^{19}$ Jun Zhu, ${ }^{20}$ Yan Chen, ${ }^{21}$ Yong Zhou, ${ }^{22}$ \\ Dong Liu, ${ }^{13}$ Weijun Pan, ${ }^{22}$ Yiyue Zhang, ${ }^{8}$ Hua Ruan, ${ }^{3}$ Feng Liu, ${ }^{23}$ Zuoyan Zhu, ${ }^{1}$ \\ Anming Meng, ${ }^{24}$ and The ZAKOC Consortium ${ }^{25}$
}

\begin{abstract}
${ }^{1}$ State Key Laboratory of Freshwater Ecology and Biotechnology, Institute of Hydrobiology, Innovation Academy for Seed Design, Chinese Academy of Sciences, Wuhan, Hubei, 430072, China; ${ }^{2}$ Key Laboratory of Cell Proliferation and Differentiation of the Ministry of Education, Peking University Genome Editing Research Center, College of Life Sciences, Peking University, Beijing, 100871, China; ${ }^{3}$ School of Life Sciences, Southwest University, Chongqing, 400715, China; ${ }^{4}$ Guangdong Medical University, Zhanjiang, Guangdong, 524023, China; ${ }^{5}$ Center for Circadian Clocks, Soochow University, Suzhou, Jiangsu, 215123, China; ${ }^{6}$ School of Life Sciences and Technology, Tongji University, Shanghai, 200092, China; ${ }^{7}$ Guangzhou Institutes of Biomedicine and Health, Chinese Academy of Sciences, Guangzhou, Guangdong, 510530, China; ${ }^{8}$ Division of Cell, Developmental and Integrative Biology, School of Medicine, South China University of Technology, Guangzhou, Guangdong, 510006, China; ${ }^{9}$ School of Medicine and Pharmacy, Ocean University of China, Qingdao, Shandong, 266100, China; ${ }^{10}$ State Key Laboratory of Neuroscience, Institute of Neuroscience, Chinese Academy of Sciences, Shanghai, 200031, China; ${ }^{11}$ Model Animal Research Center, Nanjing University, Nanjing, Jiangsu, 210093, China; ${ }^{12}$ College of Life Sciences, Zhejiang University, Hangzhou, Zhejiang, 310058, China; ${ }^{13}$ Department of Biology, South University of Science and Technology of China, Shenzhen, Guangdong, 518055, China; ${ }^{14}$ Institute of Biomedical Sciences, East China Normal University, Shanghai, 200062, China; ${ }^{15}$ College of Life Sciences, Institute of Molecular Medicine, Peking University, Beijing, 100871, China; ${ }^{16}$ School of Life Sciences, Yunnan University, Kunming, Yunnan, 650091, China; ${ }^{17}$ School of Life Sciences, Fudan University, Shanghai, 200433, China; ${ }^{18}$ State Key Laboratory of Biotherapy, West China Hospital, Sichuan University, Chengdu, Sichuan, 610041, China; ${ }^{19}$ Institutes of Brain Science, Fudan University, Shanghai, 200433, China; ${ }^{20}$ SinoFrench Research Center for Life Sciences and Genomics, Rui-jin Hospital, Shanghai Jiao Tong University School of Medicine, Shanghai, 200025, China; ${ }^{21}$ Institute of Health Sciences, Chinese Academy of Sciences \& Shanghai Jiao Tong University School of Medicine, Shanghai, 200025, China; ${ }^{22}$ CAS Key Laboratory of Nutrition, Metabolism and Food Safety, Shanghai Institute of Nutrition and Health, Chinese Academy of Sciences, Shanghai, 200031, China; ${ }^{23}$ State Key Laboratory of Membrane Biology, Institute of Zoology, Chinese Academy of Sciences, Beijing, 100101, China; ${ }^{24}$ School of Life Sciences, Tsinghua University, Beijing, 100084, China
\end{abstract}

\begin{abstract}
Genome editing by the well-established CRISPR / Cas9 technology has greatly facilitated our understanding of many biological processes. However, a complete whole-genome knockout for any species or model organism has rarely been achieved. Here, we performed a systematic knockout of all the genes (1333) on Chromosome 1 in zebrafish, successfully mutated 1029 genes, and generated 1039 germline-transmissible alleles corresponding to 636 genes. Meanwhile, by high-throughput bioinformatics analysis, we found that sequence features play pivotal roles in effective gRNA targeting at specific genes of interest, while the success rate of gene targeting positively correlates with GC content of the target sites. Moreover, we found that nearly one-fourth of all mutants are related to human diseases, and several representative CRISPR/Cas9-generated mutants are described here. Furthermore, we tried to identify the underlying mechanisms leading to distinct phenotypes between genetic mutants and antisense morpholino-mediated knockdown embryos. Altogether, this work has generated the first chromosome-wide collection of zebrafish genetic mutants by the CRISPR/Cas9 technology, which will serve as a valuable resource for the community, and our bioinformatics analysis also provides some useful guidance to design gene-specific gRNAs for successful gene editing.
\end{abstract}

[Supplemental material is available for this article.]

\footnotetext{
${ }^{25}$ A complete list of the ZAKOC Consortium authors appears at the end of this paper.

Corresponding authors: liuf@ioz.ac.cn, zyzhu@ihb.ac.cn,

mengam@mail.tsinghua.edu.cn

Article published online before print. Article, supplemental material, and publication date are at http://www.genome.org/cgi/doi/10.1101/gr.248559.119.

Freely available online through the Genome Research Open Access option.
}

C 2020 Sun et al. This article, published in Genome Research, is available under a Creative Commons License (Attribution 4.0 International), as described at http://creativecommons.org/licenses/by/4.0/. 
Zebrafish (Danio rerio) has been extensively used as a vertebrate model organism in developmental biology and genetic studies, partly due to the transparency of the early embryos and feasibility of obtaining a large quantity of progeny. In the past decades, largescale investigation of gene function in development and genetics has been achieved primarily through N-ethyl-N-nitrosourea (ENU)-mediated mutagenesis (Solnica-Krezel et al. 1994) and retroviral mutagenesis (Amsterdam et al. 1999; Golling et al. 2002), also referred to as 'forward' genetics (from phenotypes to genes) (Huang et al. 2012). Compared to a 'forward' genetic study, 'reverse' genetics (from genes to phenotypes) can precisely mutate the target sites of interest in the genome and systematically identify the mutated phenotypes (Wienholds et al. 2003; Kettleborough et al. 2013; Varshney et al. 2013). Currently, with the development and application of engineered endonucleases, including zinc finger nucleases (ZFN) (Doyon et al. 2008), transcription activator-like effector nucleases (TALEN) (Moore et al. 2012), and the CRISPR/Cas system (such as the CRISPR/Cas9 system) (Li et al. 2016; Varshney et al. 2016), the 'reverse' genetic study has evolved rapidly. The flexibility of the CRISPR/Cas9 system and data from the completed zebrafish genome sequencing project make it possible to systematically knock out every single gene in zebrafish (Howe et al. 2013; Hwang et al. 2013; Varshney et al. 2015). However, properties of gene targeting via the CRISPR/Cas9, such as the sequence bias of targetable or highly efficient sites, remain elusive and need to be substantially characterized.

To generate a comprehensive genetic resource to facilitate zebrafish research for the community, we aim to knock out all the annotated genes in zebrafish Chromosome 1, a project involving 38 laboratories from 24 institutions in China. To our knowledge, this is the first report on systematic genome targeting spanning an entire chromosome of an organism in vertebrates with the use of the CRISPR/Cas9 technology.

\section{Results}

\section{Selection of target genes on zebrafish Chromosome 1}

The 1.5-giga base-pair (Gbp) haploid nuclear genome of zebrafish comprises 25 chromosomes (Howe et al. 2013). It is estimated that there are $\sim 32,000$ genes in the genome, including $\sim 26,000$ coding genes and 2000 noncoding genes, and roughly 1300 genes per chromosome on average. The size of Chromosome 1 of zebrafish was reported to be about 60 mega base-pairs (Mbp), constituting $\sim 4 \%$ of the whole genome and containing 1418 genes (according to Zv9 release 60, 2013-01) (Table 1). Except for seven pseudogenes

Table 1. Selection of target genes on zebrafish Chromosome 1

\begin{tabular}{lrc}
\hline Gene classification & $\begin{array}{c}\text { Gene } \\
\text { counts }^{\mathbf{a}}\end{array}$ & $\begin{array}{c}\text { Selected for } \\
\text { targeting }\end{array}$ \\
\hline Pseudogene & 7 & 0 \\
Coding gene & 1202 & 1202 \\
Noncoding gene: & 99 & $21^{\mathrm{b}}$ \\
$\quad$ housekeeping & 31 & 31 \\
Noncoding gene: miRNA & 6 & 6 \\
Noncoding gene: lincRNA & 73 & 73 \\
Noncoding gene: other & 1418 & 1333 \\
\hline
\end{tabular}

${ }^{\text {a} B a s e d}$ on Zv9 release 60, 2013-01.

beventy-eight rRNA genes were excluded from our project.
Table 2. Status of zebrafish Chromosome 1 mutations

\begin{tabular}{lcc}
\hline & Gene number & Allele number \\
\hline Total tested & 1333 & \\
Fo $_{0}$ positive & 1029 & \\
F $_{1}$ positive & 636 & 1039 \\
F $_{2}$ positive & 452 & 703 \\
CZRC preserved & 491 & 693 \\
\hline
\end{tabular}

and 78 rRNA genes present on Chromosome 1, a total of 1333 genes were considered for the gene targeting attempts, consisting of 1202 coding genes and 131 noncoding genes (including 31 microRNAs and six long intergenic noncoding RNAs) (Table 1). For convenience, all of the selected target genes were numbered according to their order along Chromosome 1, with the prefix "zko." A full list of the zko genes can be found in the Supplemental Data (Supplemental Table S1).

Among these 1333 target genes (zko genes), only 109 genes had been studied with morpholino (MO) antisense oligonucleotides, 690 genes were recorded to have mutated/modified alleles in the zebrafish information network (ZFIN), an open-access online database for zebrafish research, and mutations for 606 genes had been identified from the Sanger Institute Zebrafish Mutation Project (Supplemental Table S1), while the majority of the target genes had not been subjected to mutational analysis. Furthermore, most of the mutated genes are coding genes, whereas only 39 noncoding genes had available mutations, most of which are point mutations generated through ENU mutagenesis.

\section{Summary of targeted genes/mutations on zebrafish Chromosome 1}

In order to disrupt the target genes completely, we adopted the strategy of using a single gRNA to generate indel mutations for the coding genes and a pair of gRNAs to produce genomic deletions for the noncoding genes (Supplemental Fig. S1). As to some coding genes, several gRNAs were designed and validated at the same time. Therefore, at least one gRNA for each coding gene and one pair of gRNAs for each noncoding gene was designed and tested. In rare cases, more than 10 gRNAs were designed and tested for one coding gene, such as zko187 (chtf18), since none of the 10 gRNAs gave positive results.

Up until now, 1029 (77.2\%) out of 1333 zko genes have been successfully mutated. Mutations were detected at least in founder embryos after injection of gRNA(s) together with Cas9 mRNA, including 962 coding genes and 67 noncoding genes. Mutations for 438 genes are first reported in this study. In total, 2277 gRNAs have been designed and tested, and 1086 (47.8\%) among them showed to be efficient (Supplemental Table S1). After screening the inheritance of mutant alleles, we have successfully obtained 1039 germline mutant alleles in 636 genes (61.8\% against 1029 genes), among which 703 alleles corresponding to 452 genes have given rise to the $\mathrm{F}_{2}$ generation (Table 2; Supplemental Table S2).

\section{Characterization of the features of the CRISPR/Cas9 target sequences}

It is well known that the success rate of gene targeting via the CRISPR/Cas9 system is not $100 \%$. Detectable mutations cannot be effectively induced at certain gRNA target sites, although no obvious rules could explain this phenomenon yet. To better understand the targeting capabilities of the CRISPR/Cas9 system in 
zebrafish, we analyzed the features of CRISPR/Cas9 target sequences, based on the large amounts of gRNAs tested in this study.

\section{GC-content distribution in tested target sites}

All the tested gRNA target sites were collected for the analyses of target site features, including both "positive" ones (1086 in total that gave rise to mutations in $\mathrm{F}_{0}$ fish) and "negative" ones (1191 in total that did not result in mutations in founder embryos) (Supplemental Table S1). We first compared the GC content between the two types of target sequences. The average GC content of all the tested target sites (the protospacer adjacent motif [PAM] sequence NGG was ignored, i.e., only protospacer sequences were used for the calculation) is $53.0 \%$. The slightly high GC content could be partially explained by the presence of one or two Gs at the beginning of the target sequence, which is required for an efficient in vitro transcription of gRNAs by the T7 RNA polymerase. When only considering the more important 12-nucleotide (nt) seed sequence, the average GC content for all the target sites is reduced to $50.9 \%$, close to expectation for unbiased design of target sequences. The GC content at the seed region is significantly higher in the 1086 positive target sites than in the 1191 negative ones, with $52.9 \%$, on average, for the positive sites and $49.0 \%$, on average, for the negative ones (Fig. 1A). This suggests that higher GC content in the seed region of the target site is more likely to result in successful mutation. This tendency can be seen more clearly when comparing the distribution of GC content at the seed region in the positive sites, where $70.4 \%(765 / 1086)$ of sites show GC content $>50 \%$, and that in the negative sites, where only $58.9 \%$ (703/1191) of sites show GC content $>50 \%$ (Fig. 1B). In addition, this is revealed by the strong positive correlation of the success rate of targeting with the GC content of the seed sequences (Fig. 1B,C).
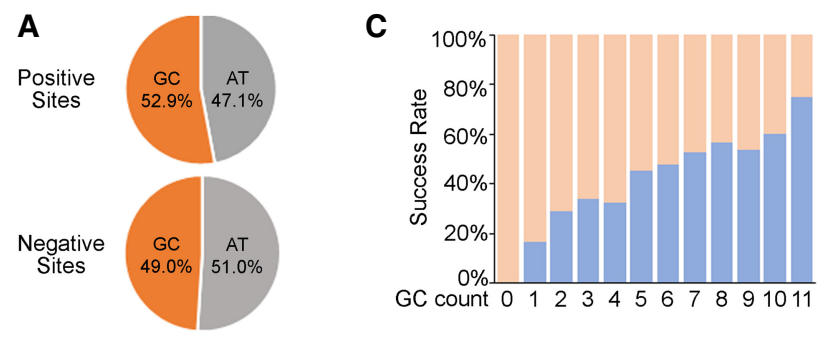

B

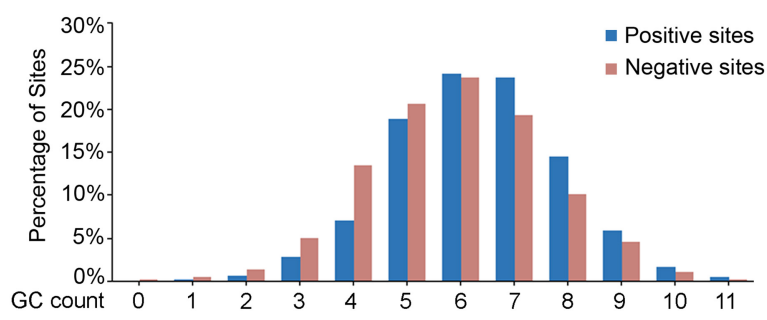

Figure 1. GC-content distribution in the 12-nt seed sequence of all the tested target sites (including 1086 positive sites and 1191 negative sites). (A) GC percentage of positive and negative target sites at the seed region, respectively. (B) GC percentage distribution of the seed region in all the tested target sites. GC count: number of $\mathrm{G}$ or $\mathrm{C}$ nucleotides in the 12-nucleotide (nt) seed sequence of the target sites. (C) Correlation of the positive rate of target sites with different GC percentages of the seed region. GC count: number of $G$ or $C$ nucleotides in the 12 -nt seed sequence of the target sites.

\section{Distribution of nucleotide motifs in tested target sites}

Positions of nucleotides and nucleotide context might influence the targeting efficiency of the CRISPR/Cas9 system. We next assessed the base composition as well as the distribution of single nucleotides and nucleotide motifs in all the tested target sites. No significant difference was detected regarding single nucleotide distribution or ratio in the first $18 \mathrm{nt}$ adjacent to the PAM region between the positive sequences and the negative ones, though there are variations among different nucleotides and at different positions (Fig. 2A; Supplemental Fig. S2), and the enrichment for G and $\mathrm{C}$ nucleotides in the positive sites is evident almost at every position (Supplemental Fig. S3). One of the few exceptions for this phenomenon is the composition of nucleotide $\mathrm{C}$ at position -1 , the first nucleotide immediately upstream of the PAM, where negative sites are dominant for this nucleotide, suggesting that $\mathrm{C}$ at this position might have a negative impact on targeting efficiency (Supplemental Fig. S3D). The significantly high percentage of nucleotide $G$ at the last two positions ( -19 and -20 relative to the PAM) is due to the obligate requirement for the presence of two terminal Gs for efficient in vitro transcription of gRNAs by T7 RNA polymerase. The distribution of PAM sequences also did not show a significant difference between the positive and negative target sites, although GGG and CGG appear slightly more frequently in the positive sites than the negative ones (Supplemental Fig. S2A).

We further compared the distribution of 2-nt and 3-nt motifs in the tested target sites and their potential relationship with mutation rate. Among all the 16 different combinations of 2-nt motifs and 64 different 3-nt motifs, we found that target sites containing motifs such as " $\mathrm{C} / \mathrm{GC} / \mathrm{G}$ " dinucleotides or " $\mathrm{C} / \mathrm{GNC} / \mathrm{G}$ " trinucleotides are more likely to induce mutations (Fig. 2B,C). This observation is consistent with our GC content analysis mentioned above as well as a previous report showing that the activity of the CRISPR/ Cas9 system is GC content-dependent (Labuhn et al. 2018).

\section{Collection and distribution of the zko alleles}

The $\mathrm{F}_{2}$ fish lines resulting from the $\mathrm{ZKO}$ project were collected and their identities were confirmed by the China Zebrafish Resource Center (CZRC) (http://en.zfish.cn) through Sanger sequencing (Supplemental Data File S1). Sperm samples were obtained from the genotype-verified fish lines and frozen for cryopreservation. In 954 confirmed mutations (Supplemental Table S2), there are 24 big indels ( $>200 \mathrm{bp}$ ) with two gRNA targeting; thus, the rest of the 930 mutant alleles were analyzed for indel characteristics. The results showed that $61.4 \%$ (571/930) are deletions with an average size of $10.9 \mathrm{bp}, 11.1 \%$ (103/930) are insertions with an average size of $6.4 \mathrm{bp}$, and 27.5\% (256/930) are indels with an average insertion of $10.3 \mathrm{bp}$ and deletion of $9 \mathrm{bp}$. In those 930 mutant alleles, $11.6 \%(108 / 930)$ are in-frame mutations and $88.4 \%(822 / 930)$ are frame-shift mutations.

A total of 693 alleles were successfully preserved by CZRC (Supplemental Table S3). All the nonsense zko alleles are listed at the websites of ZFIN (http://zfin.org/action/publication/ ZDB-PUB-171002-4/feature-list) or CZRC (http://www.zfish.cn/ TargetList.aspx). On the information page of each zko gene, the gene name, Ensembl ID, gRNA target site, target position, allele name, allele sequence, and a short description of the predicted protein product are shown. In addition, the detailed genome sequence flanking the target site, as well as a cluster analysis of the pairwise alignments (CLUSTAL) of the allele sequence and the wild-type genome sequence are presented as Portable

\section{Genome Research}

www.genome.org 
A

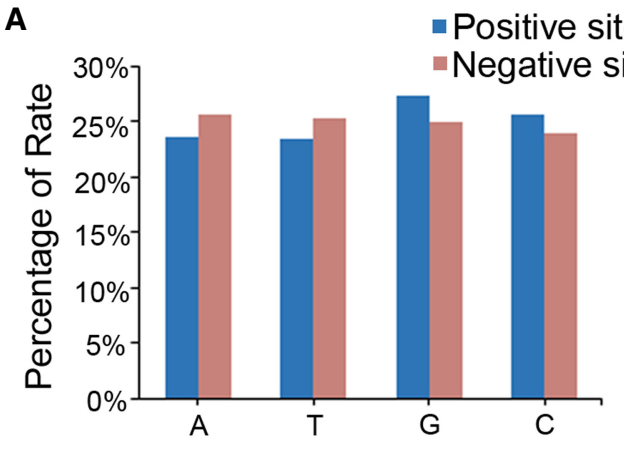

B

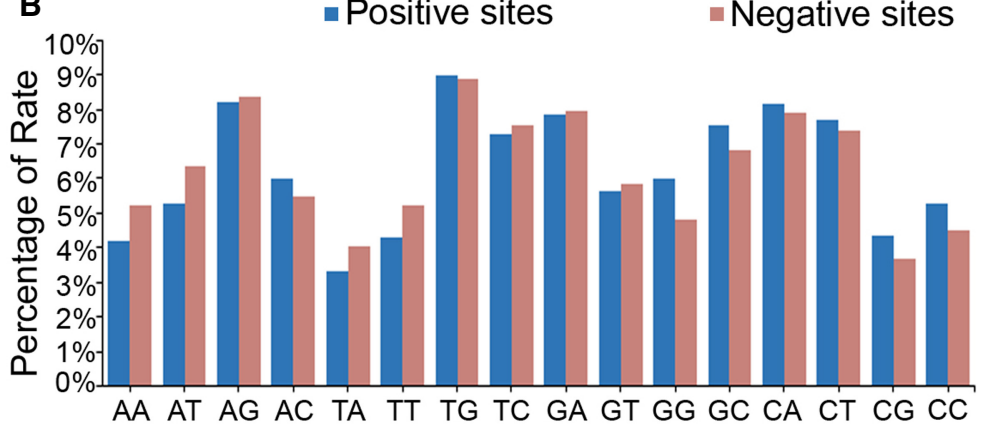

\section{C}

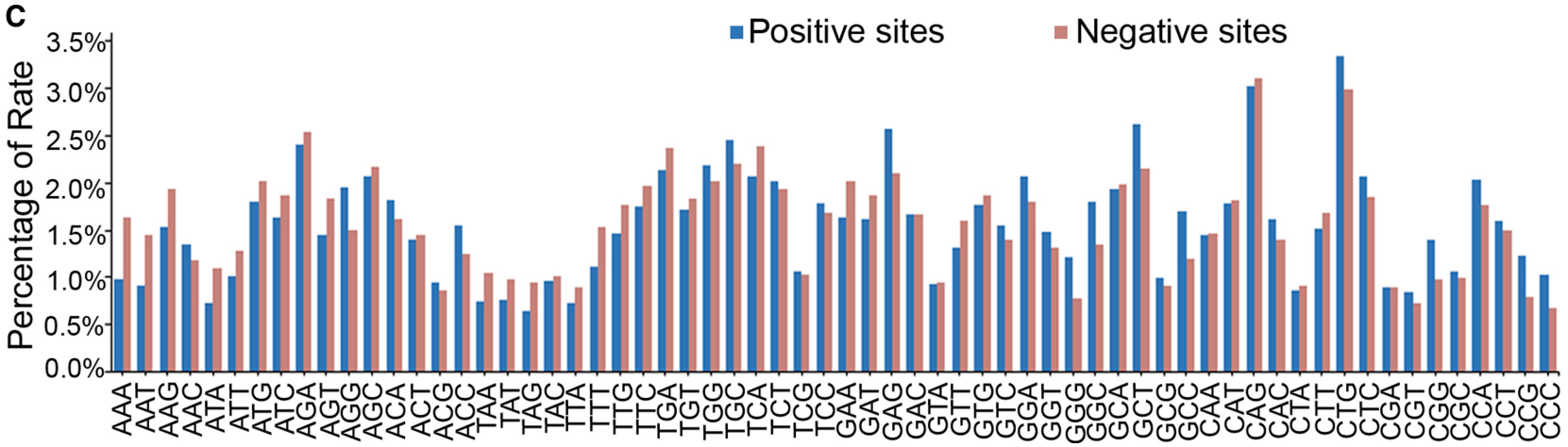

Figure 2. Distribution of nucleotide motifs in the 12-nt seed region of all the tested target sites. $(A)$ The statistical data showing the distribution of each single nucleotide in the seed region of the target sites. (B) The statistical data showing the distribution of 2-nt motifs in the seed region of the target sites. (C) The statistical data showing the distribution of 3-nt motifs in the seed region of the target sites.

Document Format (PDF) files. All the zko alleles could be ordered from CZRC through an online ordering system (http://en.zfish .cn/inforscanEN/173.html).

\section{Characterization of development- and disease-related phenotypes from the zko mutants}

We then characterized the phenotypes in the verified mutants generated using the CRISPR/Cas9 system. We exemplified the typical paradigm for detailed phenotype characterization using pleiotropic regulator 1 that is encoded by $\operatorname{plrg} 1^{z k o 487}$. plrg 1 is maternally expressed and enriched in the head and tail region at $24 \mathrm{~h}$ postfertilization (hpf) and $36 \mathrm{hpf}$ as shown by whole-mount in situ hybridization (WISH) (Fig. 3A). The homozygous plrg1 mutants with a 10-bp deletion (Fig. 3B) displayed significant developmental defects, which can be distinguished readily based on the features of black head and smaller body at $24 \mathrm{hpf}$ (Fig. 3C). To verify whether the phenotype of plrg1 is gene-specific, we designed antisense morpholinos to knock down plrg1 gene expression. The observed embryonic defects in plrg1 morphants were similar to those found in homozygous plrg1 mutants (Fig. 3C). We examined the expression of early development marker genes including gsc, ntl, and sox 17 and were unable to detect obvious defects in the homozygous plrg1 mutants until the bud stage (Fig. 3D). In contrast, the expression of hematopoietic development-related genes lmo2, gata1, and $s c l$ was decreased along with abnormal development in the plrg1 mutants (Fig. 3E). To further exclude the off-targeting effects of the CRISPR/Cas9 system, we synthesized the full-length mRNA of plrg1 to see whether we can rescue the plrg1 mutants. Our results showed that the plrg1 mRNA could rescue the early defects in the homozygous mutants, leading to their survival up to $4 \mathrm{~d}$ postfertilization (dpf), despite the apoptosis noticed (Fig. 3F,G).

Previous studies have demonstrated that Plrg1 controls cell growth by negatively regulating Tp53 class mediators (Kleinridders et al. 2009; Sorrells et al. 2012). We thus applied the tp53 MO to see whether the defects of plrg1 mutants can be alleviated. Our results showed that the mutants were efficiently rescued by injection of the $t p 53 \mathrm{MO}$ (Fig. 3H). Quantification of the embryos in different groups confirmed that the plrg1 mutants were rescued efficiently both by plrg 1 mRNA and by the tp53 MO (Fig. 3I). Moreover, genes that are essential for early zebrafish development were identified by analyzing corresponding homozygous mutants, such as two homozygous alleles of tolloid-like 1 (tll1), tll1 ${ }^{\text {zko395a }}$, and $t l^{\text {zko395b }}$, and one homozygous allele of pi4k2a 2 zko1099a 'Supplemental Fig. S4).

Among the identified mutants by the CRISPR/Cas9 system, we found that nearly one in four of the mutated genes in our study were associated with human diseases (Supplemental Table S1). For example, RUNX family transcription factor 1 (Runx1), which is encoded by runx $1^{z k o 52}$, is essential for hematopoietic stem/progenitor cell (HSPC) production in the aortagonad-mesonephros (AGM) region during embryonic hematopoiesis. Extensive studies showed that abnormal runx1 expression correlates with acute myeloid leukemia and platelet disorder (Rao 2013; Sood et al. 2017). We examined the hematopoietic phenotypes in the runx 1 mutants and found that $c-m y b$ (HSPC marker gene) expression in AGM at $36 \mathrm{hpf}$ was decreased in these mutants compared to the control embryos (Supplemental Fig. S4A), which 
A

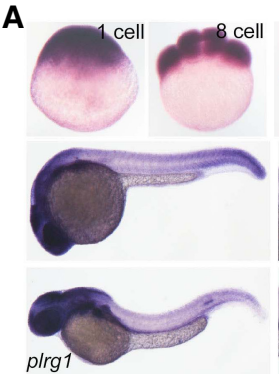

D

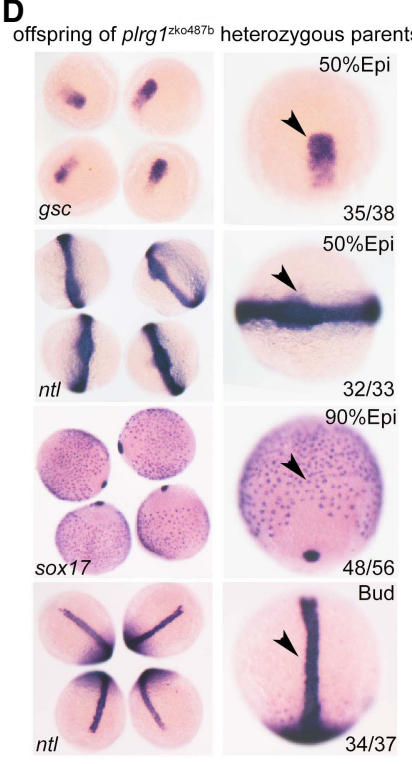

B
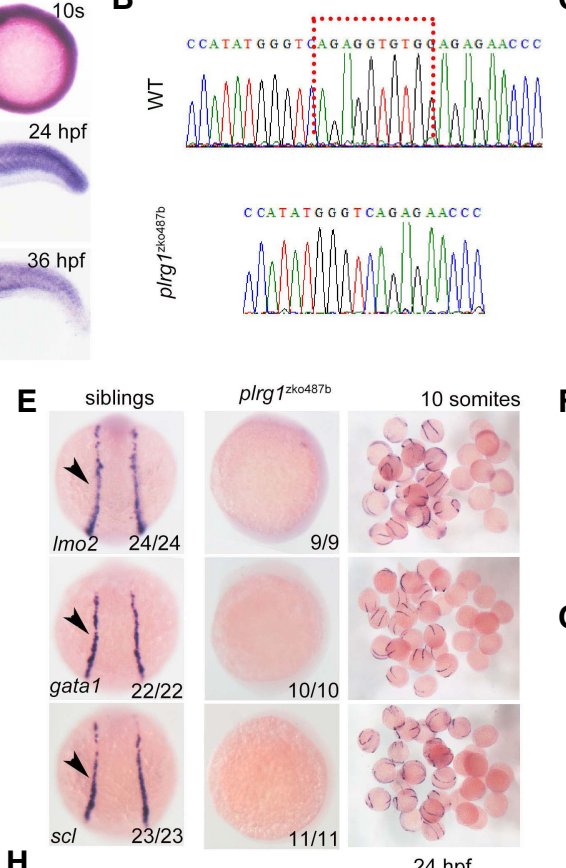

H

$11 / 1$
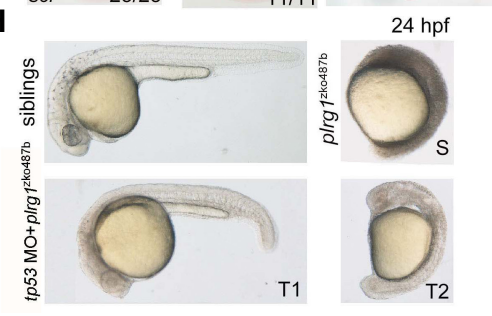

C

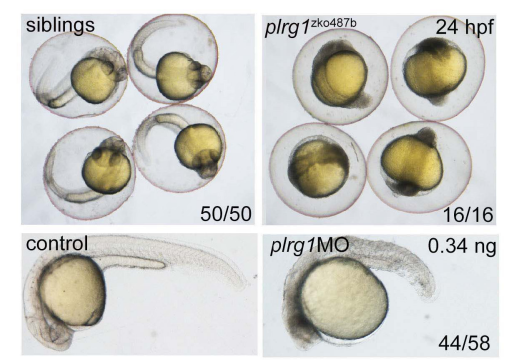

$\mathbf{F}$

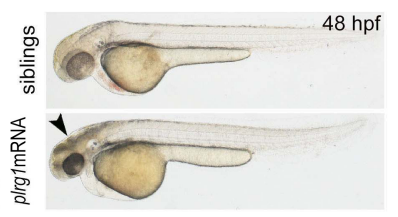

G

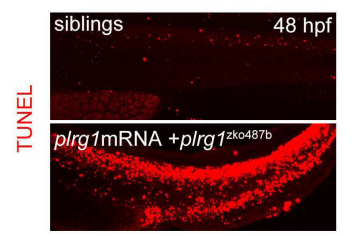

I

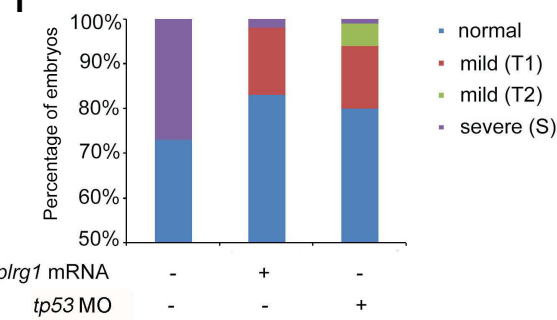

Figure 3. Characterization of $p / r g 1$ mutant generated by the CRISPR/Cas9 system. ( $A$ ) Whole-mount in situ hybridization (WISH) showing the expression of plrg1 at different developmental time points from the one-cell stage to $36 \mathrm{~h}$ postfertilization (hpf). (B) The comparison of genomic DNA sequences between wild type (WT) and plrg1 mutants with 10-base pair deletion. (C) The plrg1 mutants and morphants showed severe developmental defects, with black head and small body compared to the siblings and control embryos, respectively, at $24 \mathrm{hpf}$. (D) WISH showing the expression of gsc and $n t l$ at the $50 \%$ epiboly stage, sox 17 at $90 \%$ epiboly stage, and $n t /$ at bud stage in the offspring of plrg 1 heterozygous parents. The right panels show the magnified images, and the black arrowheads indicate corresponding expression of $g s c$ at the dorsal margin, $n t /$ at anterior axial hypoblast, forerunner cell group, and margin, and sox 17 at endoderm and forerunner cells. (E) WISH showing the expression of Imo2, gata1, and scl at lateral plate mesoderm in the siblings and plrg1 mutants. (F) Overexpression of the plrg1 full-length mRNA can rescue plrg1 mutants until $4 \mathrm{dpf}$. The body defects of mutants were rescued efficiently by mRNA overexpression, but there is still a black head at $48 \mathrm{hpf}$ (arrowhead) in plrg1 mutants. (G) TUNEL assay displays that there are obvious apoptotic signals at $48 \mathrm{hpf}$ in plrg 1 mutants injected with plrg 1 mRNA. $(H)$ Injection of tp53 morpholino can rescue the developmentally defective phenotype of plrg 1 mutants efficiently at $24 \mathrm{hpf}$. There are three subtypes of defective embryos, and we describe the siblings as normal, plrg1 mutants as severe (S), and partial rescued mutants as mild (T1) and mild (T2). (I) The quantification of plrg1 mutant embryos in different treatment groups shown in $H$.

is consistent with previously published data (Sood et al. 2010). In another example, zebrafish with $g y g 2^{z k o 624 a}$ mutation displayed increased blood glucose content (Supplemental Fig. S4B). Similarly, GYG2 mutation has been characterized as a pathogenic mutation in human Leigh syndrome, an early-onset progressive neurodegenerative disorder resulting from defective glycogen synthesis (Imagawa et al. 2014). Taken together, by following a conventional pipeline for phenotype identification of the generated mutants, we revealed a large number of previously uncharacterized phenotypes associated with development and disease.

\section{Comparison between genetic mutants generated by CRISPR / Cas9 and their corresponding morphants by antisense $\mathrm{MO}$ knockdown}

During the past two decades, reverse genetic approaches, including ZFNs, TALENs, and CRISPR/Cas9, have been developed rapidly and used extensively in genome editing and disease modeling
(Boch et al. 2009; Urnov et al. 2010; Jinek et al. 2012; Wiedenheft et al. 2012; Hwang et al. 2013). However, a large proportion of mutants generated by reverse genetics methods failed to recapitulate published morpholino-induced phenotypes (morphants) in zebrafish (Kok et al. 2015). Moreover, it was reported that genetic compensation can be induced by deleterious mutations (El-Brolosy et al. 2019; Ma et al. 2019), for example, as observed in zebrafish egfl7 mutants (Rossi et al. 2015). We also observed phenotypes inconsistent between newly generated mutants and their corresponding morphants. For instance, the mutation of $d p y 30^{z k o 989}$, encoding a histone methyltransferase complex subunit, showed no obvious phenotypes of hematopoietic cell differentiation (Supplemental Fig. S5A,B), which is inconsistent with the published morphant phenotypes that exhibit defective erythropoiesis and lymphopoiesis (Yang et al. 2014). In addition, transcriptional repressor Kruppel-like factor 3 (Klf3), which is encoded by $k l f 3^{z k o 352}$, can inhibit the expression of ferric-chelate

\section{Genome Research}

www.genome.org 
reductase $1 \mathrm{~b}$ to promote the maturation of erythroid cells in zebrafish embryos (Crossley et al. 1996; Xue et al. 2015). The homozygous klf3 mutant was able to survive until adulthood, and the maternal-zygotic klf3 mutant (hereafter MZ-klf3) showed mild erythropoiesis defects (Fig. 4A). Quantitative real-time PCR analysis showed that klf3 expression was significantly decreased in MZ$k l f 3$ at $24 \mathrm{hpf}$, whereas the expression of $k l f 1$ was up-regulated (Fig. $4 \mathrm{~B})$. These data indicate that the inconsistent phenotype between klf3 mutants and morphants could be explained by genetic compensation from other members of the Klf family in the klf3 mutants.

\section{Discussion}

In this study, we performed large-scale knockout of 1333 genes on zebrafish Chromosome 1 and successfully mutated 1029 of them. Among them, 962 are coding genes and 67 are noncoding genes. Mutations for 438 genes are first reported in this study. We also characterized the features of target sequences and revealed correlation of GC content and nucleotide motifs with the successful mutation rate (success rate) of the CRISPR/Cas9 system. At the molecular level, we reveal that the efficiency of the CRISPR/Cas9 system is highly dependent on the local sequence feature of gRNA target sites. We noticed that 304 genes have not been successfully mutated in our study, which is possibly due to the fact that they are noncoding genes or genes that are close to the telomere. In total, we generated 1039 germline-transmissible alleles corresponding to 636 genes (Supplemental Table S2), of which the information on 693 alleles corresponding to 491 genes has been shared with the ZFIN database (Supplemental Table S2). As the first step, we identified the early morphological defects in 47 of 701 alleles; the detectable phenotype rate is $6.7 \%$, such as in the plrg1 mutant and in later developmental or metabolic phenotypes in a large number of mutants, such as runx $1^{z k o 52 a}$ and $g y g 2^{z k o 624 a}$. We found that some mutants exhibit phenotypes

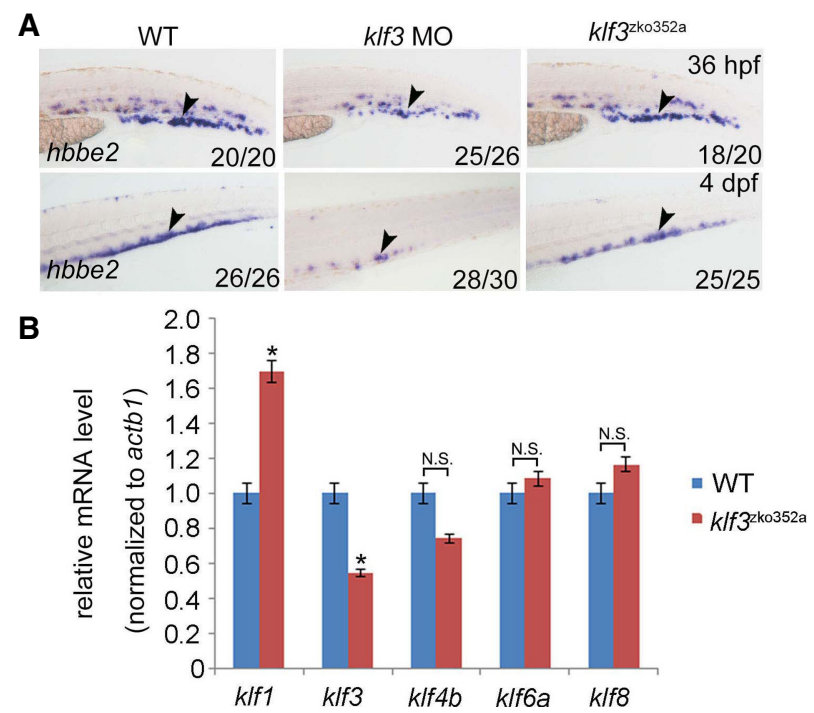

Figure 4. Phenotypic comparison between klf3 mutants and morphants. (A) WISH showing the expression of hbbe2 (also known as $\beta e 2-g l o-$ bin) in klf3 WT, morphants, and mutants. The black arrowheads indicate hbbe2 expression in erythroid lineages. (B) Quantitative real-time PCR showing the expression of Klf members klf1, klf3, klf4b, klf6a, and klf8 in klf3 mutants. inconsistent with their corresponding morphants. More importantly, we discovered that mutants of nearly one in four genes are related to human diseases.

Although there exist some differences, the overall tendencies of the sequence or mutagenesis features of the CRISPR/Cas9 target sites are largely comparable with previous publications based on zebrafish large-scale data sets. (1) Gagnon et al. observed a clear positive correlation between GC content and indel frequency in founder embryos (Gagnon et al. 2014), though Varshney et al. did not detect a significant influence of GC percentage on the mutation rate by examining the germline transmission of the mutations in founder fish (Varshney et al. 2015). We observed a strong positive correlation of the success rate of targeting with the GC content of the 12-nt seed sequences in our data set (Fig. $1)$, compatible with the result reported by Gagnon et al. (Gagnon et al. 2014). Furthermore, the positive influence of G/C nucleotides on targeting efficiencies was also supported by the observations reported by Moreno-Mateos et al., where nucleotides in the target sites were dominated by $\mathrm{G}$ or $\mathrm{C}$ enrichment, whereas $\mathrm{T}$ and A nucleotides were overall depleted (Moreno-Mateos et al. 2015). (2) We observed that the distribution of PAM sequences did not show significant differences between the positive and negative target sites, although GGG and CGG appear slightly more frequently in the positive sites than in the negative ones (Supplemental Fig. S2A), well reconciling the observation by Moreno-Mateos et al., where $\mathrm{G}$ and $\mathrm{C}$ were enriched at the first nucleotide of the PAM sequence (Moreno-Mateos et al. 2015). (3) Regarding the nucleotide at position -1 adjacent to (upstream of) the PAM sequence, our result showed that G is relatively enriched in the positive sites, while $\mathrm{C}$ is largely enriched in the negative sites (Supplemental Fig. S3C), which is consistent with the observations reported by Moreno-Mateos et al., where there is a strong enrichment for $\mathrm{G}$ but depletion in $\mathrm{C}$ at this position (Moreno-Mateos et al. 2015). This is also consistent with the observation reported by Gagnon et al., showing that target sites bearing a $G$ adjacent to the PAM motif displayed significantly higher indel frequencies than other bases (Gagnon et al. 2014).

The most valuable resource obtained from this systematic gene perturbation project is the large number of disease-mimicked mutant phenotypes. For example, a recent study reveals that the deficiency of a cilia-related gene, $p k d 2^{z k o 977 a}$, in zebrafish embryos results in phenotypes similar to those of human idiopathic scoliosis (Zhang et al. 2018). Given that it is convenient to perform highthroughput drug screening and genetic modification for disease modeling in zebrafish (Langenau and Zon 2005; Jing and Zon 2011), the abundant disease models of model organisms provide a powerful platform for preclinical trials of drug development (Zon and Peterson 2005; MacRae and Peterson 2015; Leung and Mourrain 2016).

Molecularly, it is essential to further decipher the mechanisms underlying the inconsistency in phenotypes between mutants and morphants. Our finding in klf3 mutants and morphants demonstrated that the compensatory up-regulation of $k l f 1$ is largely responsible for the phenotypic recovery in klf3 mutants. Genetic compensation (also known as transcriptional adaptation) is a common physiological phenomenon employed by organisms to accommodate genetic mutations mechanistically through DNA damage response and mutant RNA response (ElBrolosy and Stainier 2017; El-Brolosy et al. 2019; Ma et al. 2019). Additionally, it should be pointed out that maternal effects and MO off-target effects could also contribute to the phenotypic inconsistency (Kok et al. 2015; El-Brolosy and Stainier 2017). 
Taken together, the results of our study provide valuable resources for mutant phenotype identification and disease model studies in zebrafish and provide insights into the general working mechanism of the CRISPR/Cas9 system. These findings can facilitate future gRNA design in developmental biology studies and model animal-based drug discovery.

\section{Methods}

\section{Zebrafish strains}

The zebrafish strain Tuebingen was raised in automated facilities at $28.5^{\circ} \mathrm{C}$. One-cell stage embryos were used for micro-injection of Cas9 mRNA and gRNA. This study was approved by the Ethical Review Committees from the 24 institutions.

\section{In vitro synthesis of capped Cas9 mRNA and gRNA, and microinjection}

Sequences of humanized Cas9 and zebrafish-codon-optimized Cas9 were cloned into pXT7 vectors separately (Chang et al. 2013; Liu et al. 2014). Capped Cas9 mRNA was synthesized using the mMESSAGE mMACHINE mRNA transcription synthesis kit (Invitrogen, cat.\# AM1344). Then, Cas9 mRNA was purified using an RNAclean kit (TIANGEN, cat.\# DP412).

Gene-specific gRNAs were designed using the websites (http ://zifit.partners.org/ZiFiT/ and https://www.benchling.com/ crispr/). gRNAs were synthesized in vitro with a PCR product-based approach, as previously reported (Chang et al. 2013). All tested gRNAs are listed in Supplemental Table S1.

Two hundred picograms of humanized Cas9 or zebrafishcodon-optimized Cas9 mRNA and 50-100 pg of gRNA were coinjected into zebrafish embryos at the one-cell stage.

\section{Generation and identification of mutants, and phenotype observation}

Direct embryo injection with gRNAs and Cas9 mRNA generates the $\mathrm{F}_{\mathrm{O}}$ line with mosaic mutation, which usually cannot result in embryonic lethality. Further outcross of the $\mathrm{F}_{0}$ line with the wild-type line was performed to generate $F_{1}$ heterozygous lines. Finally, the $\mathrm{F}_{2}$ mutant line with homozygous mutation was obtained via incross of $F_{1}$ heterozygotes.

$\mathrm{F}_{0}$ embryos were identified by Sanger sequencing, T7E1 assay, and restriction enzyme digestion, and gRNA efficiencies were evaluated according to previous descriptions (Chang et al. 2013; Liu et al. 2014). Detailed information for heritable germline transmission strains can be found in Supplemental Table $S 2$. The $F_{1}$ or $F_{2}$ mutant lines were genotyped through Sanger sequencing of PCR fragments covering the gRNA target sites. All the frame-shifted mutants were collected by the China Zebrafish Resource Center, and the genotype of each allele was verified by Sanger sequencing.

The male and female of the $\mathrm{F}_{1}$ alleles with the same genotype were crossed to obtain $\mathrm{F}_{2}$ progeny. The $\mathrm{F}_{2}$ embryos were raised in $0.3 \times$ Danieau Buffer at $28.5^{\circ} \mathrm{C}$ and regularly observed under stereomicroscope for morphological defects before $10 \mathrm{~d}$ postfertilization.

\section{Whole-mount in situ hybridization}

Whole-mount in situ hybridization was performed as previously described (Wang et al. 2011). The Digoxigenin-labeled RNA probes detecting plrg1, gsc, ntl, sox17, lmo2, gata1, scl, hbbe2, hbbe1, cmyb, and rag1 were transcribed using T7 or SP6 polymerase.

\section{MO microinjection and mRNA overexpression}

The antisense morpholinos including plrg1 MO and tp53 MO were purchased from GeneTools. The detail sequences are shown as follows: plrg1 MO (5'-TGCTTCTGCACGTCCTCGGTCATGT-3'), tp53 MO (5' - GCGCCATTGCTTTGCAAGAATTG-3'). For injection into zebrafish embryos at the one- to four-cell stage, $0.16 \mathrm{ng}$ plrg1 $\mathrm{MO}$ and $4 \mathrm{ng}$ tp53 MO were used.

plrg1 full-length mRNA was synthesized from zebrafish cDNA using an mMESSAGE mMACHINE mRNA transcription synthesis kit (Invitrogen, cat.\# AM1344). Then, plrg1 mRNA was purified using an RNAclean kit (TIANGEN, cat.\# DP412). Seventy-five picograms of plrg1 mRNA was used for injection into zebrafish embryos at the one-cell stage.

\section{Quantitative real-time PCR}

Total RNAs from the whole embryos of siblings and mutants were extracted using TRIzol reagent (Life Technologies, cat.\# 15596018). The cDNA was reversely transcribed from the total RNAs using M-MLV Reverse Transcriptase (Promega, cat.\# M1701). Then, the cDNA was used as a template for quantitative real-time PCR.

\section{TUNEL assay}

A TUNEL assay was performed using an In Situ Death Detection kit, TMR red (Roche, cat.\# 12156792910) following the manufacturer's instructions. Briefly, plrg1 siblings and mutants at $48 \mathrm{~h}$ postfertilization were fixed with $4 \%$ paraformaldehyde and permeabilized with Proteinase $\mathrm{K}(10 \mu \mathrm{g} / \mathrm{mL})$ for $20 \mathrm{~min}$. Subsequently, the embryos were incubated with the TUNEL reaction mixture at $4^{\circ} \mathrm{C}$. After the reaction, confocal microscopy was performed using a Nikon confocal A1 laser microscope (Nikon).

\section{Data access}

All the data related to available alleles from this study have been submitted to the China Zebrafish Resource Center (CZRC) at the following link: http://www.zfish.cn/TargetList.aspx, and to the Zebrafish Information Network (ZFIN) at the following link: http ://zfin.org/action/publication/ZDB-PUB-171002-4/feature-list.

\section{ZAKOC Consortium}

Yonghua Sun, ${ }^{26}$ Luyuan Pan, ${ }^{26}$ Houpeng Wang, ${ }^{26}$ Weixun Xie, ${ }^{26}$ Mudan He, ${ }^{26}$ Ding Ye, ${ }^{26}$ Kuoyu Li, ${ }^{26}$ Feng Xiong, ${ }^{26}$ Liyue Liu, $^{26}$ Linglu $\mathrm{Li}^{26}{ }^{26}$ Yun Zhang, ${ }^{26}$ Bo Zhang, ${ }^{27}$ Da $\mathrm{Liu}^{27}$ Zhenchao Cheng, ${ }^{27}$ Yingying $\mathrm{Hu}^{27}$ Qian $\mathrm{Wu}^{27}$ Zhou Luo, ${ }^{27}$ Yutian Zhang, ${ }^{27}$ Yingdan $\mathrm{Wu}^{27}$ Wenyuan $\mathrm{Li}^{27}{ }^{27}$ Lingfei Luo, ${ }^{28}$ Jianlong $\mathrm{Ma},{ }^{28}$ Dashuang $\mathrm{Mo}^{28}$ Pengcheng Cai, ${ }^{28}$ Jinzi Chen, $^{28}$ Junhui Sun, ${ }^{28}$ Yang Zhou, ${ }^{28}$ Chuan Wu, ${ }^{28}$ Rui Ni, ${ }^{28}$ De-Li Shi, ${ }^{29,54}$ Ming Shao, ${ }^{29}$ Yan-Jun Zhang, ${ }^{29}$ Li-Jun Feng, ${ }^{29}$ XiaoNing Cheng, ${ }^{29,54}$ Ji-Tong Li, ${ }^{29}$ Yan-Yi Xing, ${ }^{29}$ Yan Zhang, ${ }^{29}$ YaPing Meng, ${ }^{29}$ Bei-Bei $\mathrm{Yu}^{29}{ }^{29}$ Han Wang, ${ }^{30}$ Jian Huang, ${ }^{30}$

\footnotetext{
${ }^{26}$ State Key Laboratory of Freshwater Ecology and Biotechnology, Institute of Hydrobiology, Innovation Academy of Seed Design, Chinese Academy of Sciences, Wuhan, Hubei, 430072, China

${ }^{27}$ Key Laboratory of Cell Proliferation and Differentiation of the Ministry of Education, Peking University Genome Editing Research Center, College of Life Sciences, Peking University, Beijing, 100871, China

${ }^{28}$ School of Life Sciences, Southwest University, Chongqing, 400715, China

${ }^{29}$ School of Life Sciences, Shandong University, Qingdao, 266237, China

${ }^{30}$ Center for Circadian Clocks, Soochow University, Suzhou, Jiangsu, 215123, China
}

\section{Genome Research}

www.genome.org 
Shuqing Zhang, ${ }^{30}$ Cheng Ji, ${ }^{30}$ Yicheng Tan, ${ }^{30}$ Jingjing Wang, ${ }^{30}$ Fanmiao Zhang, ${ }^{30}$ Mingyong Wang, ${ }^{30}$ Guodong Huang, ${ }^{30}$ Zhaomin Zhong, ${ }^{30}$ Wei Zhang, ${ }^{30}$ Zongbin Cui, ${ }^{26}$ Yong Long, ${ }^{26}$ Guili Song, ${ }^{26}$ Xiaohui $\mathrm{Li}^{26}{ }^{26} \mathrm{Xixi} \mathrm{Li}{ }^{26}$ Kai Chen, ${ }^{26}$ Jing Ren, ${ }^{26}$ Junjun Yan, ${ }^{26}$ Qing Li, ${ }^{26}$ Honghui Huang, ${ }^{28}$ Hua Ruan, ${ }^{28}$ Shicheng Zhu, ${ }^{28}$ Xuejiao Chen, ${ }^{28}$ Jingjing Pan, ${ }^{28}$ Faming Jiang, ${ }^{28}$ Jiehui Chen, ${ }^{28}$ Chao Huang, ${ }^{28}$ Guoping Dong, ${ }^{28}$ Xiaogui $\mathrm{Yi}^{28}$ Ying $\mathrm{CaO}^{31}$ Ruikun $\mathrm{Hu}^{31}$ Weilai Huang, ${ }^{31}$ Jiangfang $\mathrm{Liu}^{31}$ Hong WangXiao, ${ }^{31}$ Dong Shu, ${ }^{32}$ Jianhong Xia, ${ }^{32}$ Shaoyang Zhao, ${ }^{32}$ Pengtao Wang, ${ }^{32}$ Xiuhua $\mathrm{Wu},{ }^{32}{ }$ Wenqing Zhang, ${ }^{33}$ Yiyue Zhang, ${ }^{33}$ Mengchang $\mathrm{Xu}^{33}$ Wei Liu, ${ }^{33}$ Zhibing Huang, ${ }^{33}$ Qing Lin, ${ }^{33}$ Mei $\mathrm{Wu}^{33}$ Jianfeng Zhou, ${ }^{34}$ Aibo Sheng, ${ }^{34}$ Peipei Tang, ${ }^{34}$ Xiaoxia Gong, ${ }^{34}$ Wei Mou, ${ }^{34}$ Congcong $\mathrm{Zu}^{34}{ }^{34}$ Yun Li, ${ }^{34}$ Ling Lu, ${ }^{34}$ Yunzhang Liu, ${ }^{34}$ Xiaozhi Rong, ${ }^{34}$ Jianyang Chen, ${ }^{34}$ Jiulin Du, ${ }^{35}$ Jiwen $\mathrm{Bu}^{35}$ Xufei $\mathrm{Du},{ }^{35}$ Tingting $\mathrm{Liu}^{35}$ Shanye $\mathrm{Gu}^{35}$ Qingshun Zhao, ${ }^{36}$ Zhangji Dong, ${ }^{36}$ Xiaohua Dong, ${ }^{36}$ Shasha $\mathrm{CaO}^{36}{ }^{36}$ Yunyun Yue, ${ }^{36}$ Chun $\mathrm{Gu}^{36}$ Meijing Liu, ${ }^{36}$ Jun Chen, ${ }^{37}$ Hongjian Gong, ${ }^{37}$ Hanbing Zhong, ${ }^{38,39}$ Xuanjun Yang, ${ }^{38,39}$ Jiahao Chen, $^{38}$ Qiushi Xu, ${ }^{38}$ Tao P. Zhong, ${ }^{40}$ Daqin Jin, ${ }^{40}$ Peilu She, ${ }^{40}$ Jianjian Sun, ${ }^{40}$ Kaa Seng Lai, ${ }^{40} \mathrm{Li} \mathrm{Li},{ }^{28}$ Yu Zhang, ${ }^{28}$ Yanyan Shi, ${ }^{28}$ Fangying Zhao, ${ }^{28}$ Jing-Wei Xiong, ${ }^{41}$ Meijun Pang, ${ }^{41} \mathrm{Lu} \mathrm{Gao},{ }^{41}$ Lei Lei, ${ }^{41}$ Jinrong Peng, ${ }^{42}$ Jane Lo, ${ }^{42}$ Wuhan Xiao, ${ }^{26}$ Xiaolian Cai, ${ }^{26}$ Gang Ouyang, ${ }^{26}$ Jian Zhang, ${ }^{43,44}$ Weirui Ma, ${ }^{45}$ Liyun Miao, ${ }^{45}$ Jihua Yao, ${ }^{46} \mathrm{Yu} \mathrm{Hu},{ }^{46}$ Zhan Yin, ${ }^{26}$ Xianming Mo, ${ }^{47}$ Xue Li, ${ }^{47}$ Gang Peng, ${ }^{48}$ Cuizhen Zhang, ${ }^{48}$ Fenghua Wang, ${ }^{48}$ Jun Zhu, ${ }^{49}$ Ruimeng Yang, ${ }^{49}$ Hao Yuan, ${ }^{49}$ Yan Chen, ${ }^{50}$ Yi Pan, ${ }^{50}$ Yong Zhou, ${ }^{51}$ Dong Liu, ${ }^{38}$ Feng Zhao, ${ }^{38}$

${ }^{31}$ School of Life Sciences and Technology, Tongji University, Shanghai, 200092, China

${ }^{32}$ Guangzhou Institutes of Biomedicine and Health, Chinese Academy of Sciences, Guangzhou, Guangdong, 510530, China

${ }^{33}$ Division of Cell, Developmental and Integrative Biology, School of Medicine, South China University of Technology, Guangzhou, Guangdong, 510006, China

${ }^{34}$ School of Medicine and Pharmacy, Ocean University of China, Qingdao, Shandong, 266100, China

${ }^{35}$ State Key Laboratory of Neuroscience, Institute of Neuroscience, Chinese Academy of Sciences, Shanghai, 200031, China

${ }^{36}$ Model Animal Research Center, Nanjing University, Nanjing, Jiangsu, 210093, China

${ }^{37}$ College of Life Sciences, Zhejiang University, Hangzhou, Zhejiang, 310058, China

${ }^{38}$ Department of Biology, South University of Science and Technology of China, Shenzhen, Guangdong, 518055, China

${ }^{39}$ Institute of Chinese Medical Sciences, University of Macau, Macau, China ${ }^{40}$ Institute of Biomedical Sciences, Shanghai Key Laboratory of Regulatory Biology, East China Normal University, Shanghai, 200062, China

${ }^{41}$ College of Life Sciences, Institute of Molecular Medicine, Peking University, Beijing, 100871, China

${ }^{42}$ College of Animal Sciences, Zhejiang University, Hangzhou, Zhejiang, 310058, China

${ }^{43}$ State Key Laboratory for Conservation and Utilization of Bio-Resources, Kunming, 650091, China

${ }^{44}$ Center for Life Sciences, School of Life Sciences, Yunnan University, Kunming, 650091, China

${ }^{45}$ State Key Laboratory of Molecular Developmental Biology, Institute of Genetics and Developmental Biology, Chinese Academy of Sciences, Beijing, 100101, China

${ }^{46}$ School of Life Sciences, Fudan University, Shanghai, 200433, China

${ }^{47}$ State Key Laboratory of Biotherapy, West China Hospital, Sichuan University, Chengdu, Sichuan, 610041, China

${ }^{48}$ Institutes of Brain Science, Fudan University, Shanghai, 200433, China

${ }^{49}$ Sino-French Research Center for Life Sciences and Genomics, Rui-Jin Hospital, Shanghai Jiao Tong University School of Medicine, Shanghai, 200025, China

${ }^{50}$ Institute of Health Sciences, Chinese Academy of Sciences \& Shanghai Jiao Tong University School of Medicine, Shanghai, 200025, China

${ }^{51}$ CAS Key Laboratory of Nutrition, Metabolism and Food Safety, Shanghai Institute of Nutrition and Health, Chinese Academy of Sciences, Shanghai, 200031, China
Weijun Pan, ${ }^{50}$ Feng Liu, ${ }^{52}$ Yanyan Ding, ${ }^{52}$ Yuanyuan Xue, ${ }^{52}$ $\mathrm{Lu}$ Wang, ${ }^{52}$ Yifan Zhang, ${ }^{52}$ Dongyuan $\mathrm{Ma}^{52}$ Xinyan $\mathrm{Lu}^{52}$ Shuai Gao, ${ }^{52}$ Jun Xia, ${ }^{52}$ Zuoyan Zhu, ${ }^{26}$ Anming Meng, ${ }^{53}$ Xingfeng $\mathrm{Liu}^{53}$ Juhui $\mathrm{Qiu}^{53}$ Bo Gong, ${ }^{53}$ Luxi Chen, ${ }^{53}$ Cong Xiong, ${ }^{53}$ Likun Yao, ${ }^{53}$ Cencan Xing, ${ }^{53}$ Yixia Wang, ${ }^{53}$ Weimin Shen, ${ }^{53}$ and Jiawei Sun ${ }^{53}$

\section{Acknowledgments}

We thank all members of ZAKOC (Zebrafish All Genes KO Consortium for Chromosome 1) for the efforts contributed to this work. This work was supported by grants from the Ministry of Science and Technology of China (2018YFA0801000, 2018YFA0800200), the National Natural Science Foundation of China (31830061, 31721005), and the Strategic Priority Research Program of the Chinese Academy of Sciences, China.

Author contributions: F.L., Y.S., Z.Z., and A.M. conceived the project, analyzed the data, and wrote the paper with the help of other members of ZAKOC. All authors read and approved the final manuscript.

\section{References}

Amsterdam A, Burgess S, Golling G, Chen W, Sun Z, Townsend K, Farrington S, Haldi M, Hopkins N. 1999. A large-scale insertional mutagenesis screen in zebrafish. Genes Dev 13: 2713-2724. doi:10.1101/gad .13.20.2713

Boch J, Scholze H, Schornack S, Landgraf A, Hahn S, Kay S, Lahaye T, Nickstadt A, Bonas U. 2009. Breaking the code of DNA binding specificity of TAL-type III effectors. Science 326: 1509-1512. doi:10.1126/sci ence. 1178811

Chang N, Sun C, Gao L, Zhu D, Xu X, Zhu X, Xiong JW, Xi JJ. 2013. Genome editing with RNA-guided Cas9 nuclease in Zebrafish embryos. Cell Res 23: 465-472. doi:10.1038/cr.2013.45

Crossley M, Whitelaw E, Perkins A, Williams G, Fujiwara Y, Orkin SH. 1996. Isolation and characterization of the cDNA encoding BKLF/TEF-2, a major CACCC-box-binding protein in erythroid cells and selected other cells. Mol Cell Biol 16: 1695-1705. doi:10.1128/MCB.16.4.1695

Doyon Y, McCammon JM, Miller JC, Faraji F, Ngo C, Katibah GE, Amora R, Hocking TD, Zhang L, Rebar EJ, et al. 2008. Heritable targeted gene disruption in zebrafish using designed zinc-finger nucleases. Nat Biotechnol 26: 702-708. doi:10.1038/nbt1409

El-Brolosy MA, Stainier DYR. 2017. Genetic compensation: a phenomenon in search of mechanisms. PLoS Genet 13: e1006780. doi:10.1371/jour nal.pgen.1006780

El-Brolosy MA, Kontarakis Z, Rossi A, Kuenne C, Günther S, Fukuda N, Kikhi K, Boezio GLM, Takacs CM, Lai SL, et al. 2019. Genetic compensation triggered by mutant mRNA degradation. Nature 568: 193-197. doi:10 .1038/s41586-019-1064-Z

Gagnon JA, Valen E, Thyme SB, Huang P, Akhmetova L, Pauli A, Montague TG, Zimmerman S, Richter C, Schier AF. 2014. Efficient mutagenesis by Cas 9 protein-mediated oligonucleotide insertion and large-scale assessment of single-guide RNAs. PLoS One 9: e98186. doi:10.1371/journal .pone.0098186

Golling G, Amsterdam A, Sun Z, Antonelli M, Maldonado E, Chen W, Burgess S, Haldi M, Artzt K, Farrington S, et al. 2002. Insertional mutagenesis in zebrafish rapidly identifies genes essential for early vertebrate development. Nat Genet 31: 135-140. doi:10.1038/ng896

Howe K, Clark MD, Torroja CF, Torrance J, Berthelot C, Muffato M, Collins JE, Humphray S, McLaren K, Matthews L, et al. 2013. The zebrafish reference genome sequence and its relationship to the human genome. Nature 496: 498-503. doi:10.1038/nature12111

Huang P, Zhu Z, Lin S, Zhang B. 2012. Reverse genetic approaches in zebrafish. J Genet Genomics 39: 421-433. doi:10.1016/j.jgg.2012.07.004

Hwang WY, Fu Y, Reyon D, Maeder ML, Tsai SQ, Sander JD, Peterson RT, Yeh JR, Joung JK. 2013. Efficient genome editing in zebrafish using a CRISPR-Cas system. Nat Biotechnol 31: 227-229. doi:10.1038/nbt.2501

\footnotetext{
${ }^{52}$ State Key Laboratory of Membrane Biology, Institute of Zoology, Chinese Academy of Sciences, Beijing, 100101, China

${ }^{5}$ School of Life Sciences, Tsinghua University, Beijing, 100084, China

${ }^{54}$ Present address: Affiliated Hospital of Guangdong Medical University, Zhanjiang, 524001, China
} 
Imagawa E, Osaka H, Yamashita A, Shiina M, Takahashi E, Sugie H, Nakashima M, Tsurusaki Y, Saitsu H, Ogata K, et al. 2014. A hemizygous GYG2 mutation and Leigh syndrome: a possible link? Hum Genet 133: 225-234. doi:10.1007/s00439-013-1372-6

Jinek M, Chylinski K, Fonfara I, Hauer M, Doudna JA, Charpentier E. 2012. A programmable dual-RNA-guided DNA endonuclease in adaptive bacterial immunity. Science 337: 816-821. doi:10.1126/science.1225829

Jing L, Zon LI. 2011. Zebrafish as a model for normal and malignant hematopoiesis. Dis Model Mech 4: 433-438. doi:10.1242/dmm.006791

Kettleborough RN, Busch-Nentwich EM, Harvey SA, Dooley CM, de Bruijn E, van Eeden F, Sealy I, White RJ, Herd C, Nijman IJ, et al. 2013. A systematic genome-wide analysis of zebrafish protein-coding gene function. Nature 496: 494-497. doi:10.1038/nature11992

Kleinridders A, Pogoda HM, Irlenbusch S, Smyth N, Koncz C, Hammerschmidt M, Bruning JC. 2009. PLRG1 is an essential regulator of cell proliferation and apoptosis during vertebrate development and tissue homeostasis. Mol Cell Biol 29: 3173-3185. doi:10.1128/MCB .01807-08

Kok FO, Shin M, Ni CW, Gupta A, Grosse AS, van Impel A, Kirchmaier BC, Peterson-Maduro J, Kourkoulis G, Male I, et al. 2015. Reverse genetic screening reveals poor correlation between morpholino-induced and mutant phenotypes in zebrafish. Dev Cell 32: 97-108. doi:10.1016/j .devcel.2014.11.018

Labuhn M, Adams FF, Ng M, Knoess S, Schambach A, Charpentier EM, Schwarzer A, Mateo JL, Klusmann JH, Heckl D. 2018. Refined sgRNA efficacy prediction improves large- and small-scale CRISPR-Cas9 applications. Nucleic Acids Res 46: 1375-1385. doi:10.1093/nar/gkx1268

Langenau DM, Zon LI. 2005. The zebrafish: a new model of T-cell and thymic development. Nat Rev Immunol 5: 307-317. doi:10.1038/nri1590

Leung LC, Mourrain P. 2016. Drug discovery: zebrafish uncover novel antipsychotics. Nat Chem Biol 12: 468-469. doi:10.1038/nchembio.2114

Li M, Zhao L, Page-McCaw PS, Chen W. 2016. Zebrafish genome engineering using the CRISPR-Cas9 system. Trends Genet 32: 815-827. doi:10 .1016/j.tig.2016.10.005

Liu D, Wang Z, Xiao A, Zhang Y, Li W, Zu Y, Yao S, Lin S, Zhang B. 2014. Efficient gene targeting in zebrafish mediated by a zebrafish-codon-optimized Cas9 and evaluation of off-targeting effect. J Genet Genomics 41: 43-46. doi:10.1016/j.jgg.2013.11.004

Ma Z, Zhu P, Shi H, Guo L, Zhang Q, Chen Y, Chen S, Zhang Z, Peng J, Chen J. 2019. PTC-bearing mRNA elicits a genetic compensation response via Upf3a and COMPASS components. Nature 568: 259-263. doi:10.1038/ s41586-019-1057-y

MacRae CA, Peterson RT. 2015. Zebrafish as tools for drug discovery. Nat Rev Drug Discov 14: 721-731. doi:10.1038/nrd4627

Moore FE, Reyon D, Sander JD, Martinez SA, Blackburn JS, Khayter C, Ramirez CL, Joung JK, Langenau DM. 2012. Improved somatic mutagenesis in zebrafish using Transcription Activator-Like Effector Nucleases (TALENs). PLoS One 7: e37877. doi:10.1371/journal.pone .0037877

Moreno-Mateos MA, Vejnar CE, Beaudoin JD, Fernandez JP, Mis EK, Khokha MK, Giraldez AJ. 2015. CRISPRscan: designing highly efficient sgRNAs for CRISPR-Cas9 targeting in vivo. Nat Methods 12: 982-988. doi:10 $.1038 /$ nmeth.3543

Rao AK. 2013. Spotlight on FLI1, RUNX1, and platelet dysfunction. Blood 122: 4004-4006. doi:10.1182/blood-2013-10-533166
Rossi A, Kontarakis Z, Gerri C, Nolte H, Hölper S, Krüger M, Stainier DY. 2015. Genetic compensation induced by deleterious mutations but not gene knockdowns. Nature 524: 230-233. doi:10.1038/nature14580

Solnica-Krezel L, Schier AF, Driever W. 1994. Efficient recovery of ENU-induced mutations from the zebrafish germline. Genetics 136: 1401-1420.

Sood R, English MA, Belele CL, Jin H, Bishop K, Haskins R, McKinney MC, Chahal J, Weinstein BM, Wen Z, et al. 2010. Development of multilineage adult hematopoiesis in the zebrafish with a runx 1 truncation mutation. Blood 115: 2806-2809. doi:10.1182/blood-2009-08-236729

Sood R, Kamikubo Y, Liu P. 2017. Role of RUNX1 in hematological malignancies. Blood 129: 2070-2082. doi:10.1182/blood-2016-10-687830

Sorrells S, Carbonneau S, Harrington E, Chen AT, Hast B, Milash B, Pyati U, Major MB, Zhou Y, Zon LI, et al. 2012. Ccdc94 protects cells from ionizing radiation by inhibiting the expression of p53. PLoS Genet 8: e1002922. doi:10.1371/journal.pgen.1002922

Urnov FD, Rebar EJ, Holmes MC, Zhang HS, Gregory PD. 2010. Genome editing with engineered zinc finger nucleases. Nat Rev Genet 11: 636-646. doi:10.1038/nrg2842

Varshney GK, Lu J, Gildea DE, Huang H, Pei W, Yang Z, Huang SC, Schoenfeld D, Pho NH, Casero D, et al. 2013. A large-scale zebrafish gene knockout resource for the genome-wide study of gene function. Genome Res 23: 727-735. doi:10.1101/gr.151464.112

Varshney GK, Pei W, LaFave MC, Idol J, Xu L, Gallardo V, Carrington B, Bishop K, Jones M, Li M, et al. 2015. High-throughput gene targeting and phenotyping in zebrafish using CRISPR/Cas9. Genome Res 25: 1030-1042. doi:10.1101/gr.186379.114

Varshney GK, Carrington B, Pei W, Bishop K, Chen Z, Fan C, Xu L, Jones M, LaFave MC, Ledin J, et al. 2016. A high-throughput functional genomics workflow based on CRISPR/Cas9-mediated targeted mutagenesis in zebrafish. Nat Protoc 11: 2357-2375. doi:10.1038/nprot.2016.141

Wang L, Zhang PP, Wei YL, Gao Y, Patient R, Liu F. 2011. A blood flow-dependent klf2a-NO signaling cascade is required for stabilization of hematopoietic stem cell programming in zebrafish embryos. Blood 118: 4102-4110. doi:10.1182/blood-2011-05-353235

Wiedenheft B, Sternberg SH, Doudna JA. 2012. RNA-guided genetic silencing systems in bacteria and archaea. Nature 482: 331-338. doi:10.1038/ nature 10886

Wienholds E, van Eeden F, Kosters M, Mudde J, Plasterk RH, Cuppen E. 2003. Efficient target-selected mutagenesis in zebrafish. Genome Res 13: 2700-2707. doi:10.1101/gr.1725103

Xue Y, Gao S, Liu F. 2015. Genome-wide analysis of the zebrafish Klf family identifies two genes important for erythroid maturation. Dev Biol 403: 115-127. doi:10.1016/j.ydbio.2015.05.015

Yang Z, Augustin J, Chang C, Hu J, Shah K, Chang CW, Townes T, Jiang H. 2014. The DPY30 subunit in SET1/MLL complexes regulates the proliferation and differentiation of hematopoietic progenitor cells. Blood 124: 2025-2033. doi:10.1182/blood-2014-01-549220

Zhang X, Jia S, Chen Z, Chong YL, Xie H, Feng D, Wu X, Song DZ, Roy S, Zhao C. 2018. Cilia-driven cerebrospinal fluid flow directs expression of urotensin neuropeptides to straighten the vertebrate body axis. Nat Genet 50: 1666-1673. doi:10.1038/s41588-018-0260-3

Zon LI, Peterson RT. 2005. In vivo drug discovery in the zebrafish. Nat Rev Drug Discov 4: 35-44. doi:10.1038/nrd1606

Received January 22, 2019; accepted in revised form November 6, 2019.

\section{Genome Research}

www.genome.org 


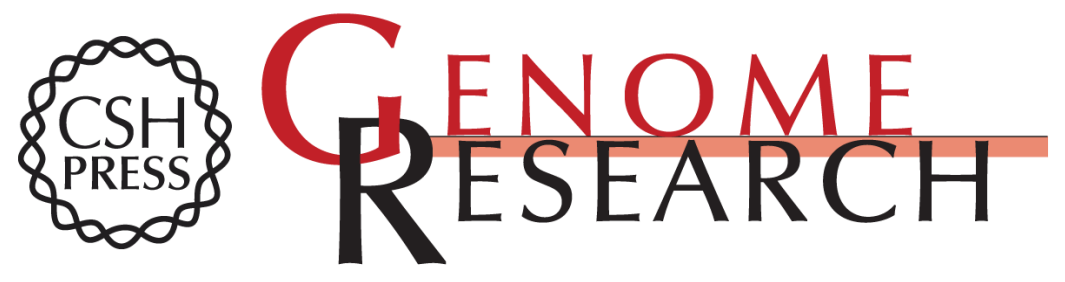

\section{Systematic genome editing of the genes on zebrafish Chromosome 1 by CRISPR/Cas9}

Yonghua Sun, Bo Zhang, Lingfei Luo, et al.

Genome Res. 2020 30: 118-126 originally published online December 12, 2019

Access the most recent version at doi:10.1101/gr.248559.119

Supplemental Material

References

Open Access

Creative Commons

License

Email Alerting Service
http://genome.cshlp.org/content/suppl/2019/12/12/gr.248559.119.DC1

This article cites 44 articles, 15 of which can be accessed free at: http://genome.cshlp.org/content/30/1/118.full.html\#ref-list-1

Freely available online through the Genome Research Open Access option.

This article, published in Genome Research, is available under a Creative Commons License (Attribution 4.0 International), as described at http://creativecommons.org/licenses/by/4.0/.

Receive free email alerts when new articles cite this article - sign up in the box at the top right corner of the article or click here.

\section{Affordable, Accurate Sequencing.}

